\title{
Perfil da composição química e atividades antibacteriana e antioxidante do óleo essencial do cravo-da-índia (Eugenia caryophyllata Thunb.)
}

\author{
Jandimara Doninelli Fior Silvestri ${ }^{1}$, Natalia Paroul ${ }^{2}$, Eliane Czyewski ${ }^{1}$, Lindomar Lerin ${ }^{3}$, Ieda Rotava ${ }^{3}$, Rogério \\ Luis Cansian ${ }^{4}$, Altemir Mossi ${ }^{4}$, Geciane Toniazzo ${ }^{5}$, Débora de Oliveira ${ }^{5}$, Helen Treichel ${ }^{*}$
}

\begin{abstract}
A busca por substâncias antibacterianas e antioxidantes naturais tem sido alvo de diversos estudos científicos. Foi objetivo determinar as atividades antibacteriana e antioxidante e a concentração inibitória mínima do óleo essencial do cravo-da-índia, obtido por hidrodestilação. O estudo por CG-EM identificou o eugenol como o constituinte majoritário do óleo essencial e os testes de atividade antibacteriana mostraram bons resultados para quase todos os microorganismos avaliados. Os valores de concentração inibitória mínima variaram de 0,2 mg.mL $\mathrm{mL}^{-1}$ a 0,6 mg.mL ${ }^{-1}$. A atividade antioxidante demonstrou uma correlação linear com a concentração de óleo essencial. Os resultados demonstraram potencial antibacteriano e antioxidante do óleo essencial de Eugenia caryophyllata, constituindo-se uma opção para a formulação de novos produtos alimentícios.
\end{abstract}

Palavras-chave: Atividade antibacteriana, atividade antioxidante, Eugenia caryophyllata.

\section{RESUMO}

\section{Chemical composition and antioxidant and antibacterial activities of clove essential oil (Eugenia caryophyllata Thunb)}

The search for natural antioxidants and antimicrobials has been a major goal of scientific studies. The main objective of this work was to determine the antioxidant and antimicrobial activities and the minimal inhibitory concentration of clove oil obtained by hydrodistillation. GC-MS analysis identified eugenol as major compound of the essential oil. The oil showed antibacterial activity for almost all tested microorganisms. The minimal inhibitory concentration ranged from $0.2 \mathrm{mg} \cdot \mathrm{mL}^{-1}$ to $0.6 \mathrm{mg} \cdot \mathrm{mL}^{-1}$. There was a linear correlation between the antioxidant activity and the essential oil concentration. Results showed the potential of Eugenia caryophyllata essential oil as an alternative for the formulation of new food products.

Key words: Antimicrobial activity, antioxidant activity, Eugenia caryophyllata Thunb.

Recebido para publicação em abril de 2009 e aprovado em agosto de 2010

${ }^{1}$ Químicas, Graduandas. Departamento de Química, Universidade Regional Integrada - Campus de Erechim, Av. Sete de Setembro 1621, 99700-000, Erechim, Rio Grande do Sul, Brasil. jandi.silvestri@gmail.com; eliane_czyewski@hotmail.com

${ }^{2}$ Química, Mestre. Departamento de Química, Universidade Regional Integrada - Campus de Erechim, Av. Sete de Setembro 1621, 99700-000, Erechim, Rio Grande do Sul, Brasil. nparoul@uricer.edu.br

${ }^{3}$ Biólogos, Doutores. Instituto de Química, Universidade Federal do Rio de Janeiro, Centro de Tecnologia, Bloco A, 21945-900, Rio de Janeiro, Rio de Janeiro, Brasil. lindolerin@gmail.com; iedarottava@gmail.com

${ }^{4}$ Engenheiros-Agrônomos, Doutores. Universidade Regional Integrada - Campus de Erechim, Av. Sete de Setembro 1621, 99700-000, Erechim, Rio Grande do Sul, Brasil. cansian@uricer.edu.br; amossi@uricer.edu.br

${ }^{5}$ Engenheiras de Alimentos, Doutoras. Departamento de Engenharia de Alimentos, Universidade Regional Integrada - Campus de Erechim, Av. Sete de Setembro 1621, 99700-000, Erechim, Rio Grande do Sul, Brasil. gtoniazzo@uricer.edu.br; odebora@uricer.edu.br; *helen@uricer.edu.br (autor para correspondência) 


\section{INTRODUÇÃO}

Os alimentos de origem animal ou vegetal, frescos ou processados, podem veicular diversos micro-organismos patogênicos causadores de doenças (Souza et al., 2003), representando um importante problema de saúde pública e de economia para a sociedade (Celiktas et al., 2007).

A presença de micro-organismos patogênicos e suas toxinas, principalmente patógenos como Escherichia coli O157:H7 (Moreira et al., 2005), causa sérios problemas à saúde dos consumidores, além de diminuir a vida útil dos alimentos, gerando perdas econômicas (Souza et al., 2003). Além disso, o uso de conservantes sintéticos, muitos deles com atividade carcinogênica e teratogênica, tem diminuído sua aceitação pelos consumidores (Nascimento et al., 2000; Moreira et al., 2005).

Neste sentido, a utilização de plantas aromáticas, possuidoras de óleos essenciais, geralmente com ações flavorizantes, com comprovada ação antibacteriana e antioxidante, pode ser uma alternativa interessante para a conservação de alimentos, diminuindo a concentração de aditivos sintéticos nesses produtos.

Pesquisas têm citado as propriedades antibacterianas e antifúngicas das especiarias in natura, seus óleos essenciais e seus extratos. Várias plantas usadas para aromatizar alimentos são apontadas por apresentarem atividade antimicrobiana como, por exemplo, louro (Laurus nobilis L.), manjerona (Origanum majorana L.), manjericão (Ocimum basilicum L.), cravo (Eugenia caryophyllata), canela (Cinnamomum zeylanicum Blume), coentro (Coriandrum sativum L.), melissa (Melissa officinalis L.), limão (Citrus aurantiifolia (Christm.) Swingle), laranja (Citrus aurantium L.), alecrim (Rosmarinus officinalis L.), menta (Mentha piperita L.), alho (Allium sativum L.) entre outras (Celiktas et al., 2007).

Por outro lado, a oxidação lipídica, uma das principais causas de deterioração de alimentos, pode ser prevenida pela ação de antioxidantes, dos quais os sintéticos, BHA (butil hidroxianisol), BHT (butil hidroxitolueno), PG (propil galato) e TBHQ (terc-butil hidroquinoma) são os mais utilizados. O emprego destes compostos, entretanto, tem sido questionado quanto a sua toxicidade, motivando a busca por novos antioxidantes que possam atuar isolados ou sinergicamente com outros aditivos, em substituição aos sintéticos (Semwal et al., 1997).

A procura por novos agentes antimicrobianos e antioxidantes, a partir de plantas, para uso nas indústrias alimentícias, é intensa, em decorrência da crescente resistência dos micro-organismos patogênicos aos produtos sintéticos (Tepe et al., 2004).
O cravo-da-índia (Eugenia caryophyllata Thunb.) é uma planta arbórea, nativa das Ilhas Molucas (Arquipélago da Insulíndia, Indonésia), possui odor fortemente aromático, sabor ardente e característico. Das sementes, de aroma ativo, extrai-se o ácido eugênico, incolor e de sabor picante. Sua composição química é constituída principalmente por eugenol, acetato de eugenol, betacariofileno, ácido oleânico, e substâncias das classes: triterpeno, ceras vegetais, cetonas, resinas, taninos e esteróis. O eugenol apresenta efeito anti-inflamatório, cicatrizante, analgésico e é eficaz no combate e diminuição de bactérias presentes na boca. Seus efeitos medicinais compreendem o tratamento de náuseas, flatulências, indigestão, diarreia. Com propriedades antibactericidas é também usado como anestésico e antisséptico para o alívio de dores de dente (Nascimento et al., 2000). Além de seus efeitos nessas aplicações, os extratos de cravo-daíndia reduzem o número de Escherichia coli e outras bactérias durante a armazenagem de sucos, leites e chás (Mau et al., 2001).

Embora haja diversos trabalhos com extratos de cravo-da-índia, existe uma lacuna na literatura em relação à avaliação do potencial antimicrobiano e antioxidante desse óleo essencial, visando ao seu aproveitamento na indústria alimentícia, tendo sido este o objetivo principal do presente trabalho.

\section{MATERIAL E MÉTODOS}

\section{Obtenção e composição química do óleo essencial do cravo-da-índia}

O óleo essencial foi obtido a partir de uma amostra comercial de cravo-da-índia, desidratado, adquirida em mercado local em Erechim, Rio Grande do Sul, Brasil.

A extração do óleo essencial foi realizada por hidrodestilação em aparelho tipo Clevenger, utilizando-se $200 \mathrm{~g}$ de amostra em 600 mL de água destilada por 90 minutos após o início da ebulição.

A análise da composição química do óleo essencial de cravo-da-índia foi realizada por cromatografia gasosa, acoplada à espectrometria de massas (CG-EM), em equipamento Shimadzu, Modelo QP 5050A. A amostra utilizada na cromatografia foi preparada a $5000 \mathrm{mg} . \mathrm{L}^{-1}$, sendo o óleo dissolvido em diclorometano (Merck). Foi empregada uma coluna capilar DB-5 (30 m x 0,25 mm de diâmetro x 0,25 $\mu \mathrm{m}$ de espessura do filme); vazão do gás de arraste (hélio) de 0,8 $\mathrm{mL} / \mathrm{min}$; detector em 1,0 Kv; Modo split (1:20); injetor a $280^{\circ} \mathrm{C}$ e interface em $300^{\circ} \mathrm{C}$. Programação da temperatura inicial $50^{\circ} \mathrm{C}$ ( $3 \mathrm{~min}$ ); rampa de aquecimento: $4^{\circ} \mathrm{C} / \mathrm{min}$ até 300 ${ }^{\circ} \mathrm{C}$ e um tempo de corte do solvente de $4 \mathrm{~min}$. O tempo total de análise foi de 65,5 minutos, utilizando $70 \mathrm{eV}$. Os espectros de massa dos compostos foram comparados com o banco de dados (Wiley) existente no equipamento. 


\section{Atividade antibacteriana}

Os testes antimicrobianos foram realizados por duas metodologias: a de difusão em placas e a do método indireto de crescimento bacteriano (Maloz, 2005).

Foram selecionados dezoito micro-organismos para a análise da atividade antibacteriana, sendo eles bactérias Gram-positivas (Enterococcus faecalis, Bacillus subtilis, Micrococcus luteus, Sarcina sp., Staphylococcus aureus, Staphylococcus epidermidis, Streptococcus mutans) e Gram-negativas (Acinetobacter sp., Aeromonas sp., Citrobacter freundii, Enterobacter cloacae, Escherichia coli, Proteus vulgaris, Pseudomonas aeruginosa, Salmonella choleraesuis, Serratia marcescens, Shigella flexneri, Xanthomonas campestris), crescidos previamente em meio Lúria Bentani (10 g/L de triptona, 5 g/L de extrato de levedura e $5 \mathrm{~g} / \mathrm{L}$ de $\mathrm{NaCl}$ ) durante 24 horas a $36 \pm 1^{\circ} \mathrm{C}$.

A primeira metodologia foi executada em placas de Petri, com meio de cultura Ágar Müeller-Hinton e discos de papel Whatmann 3 com $7 \mathrm{~mm}$ de diâmetro. As culturas ativas das bactérias foram inoculadas por espalhamento nas placas num volume de 200 ì $\left(10^{8}\right.$ UFC.mL $\left.{ }^{-1}\right)$. Para todas as bactérias testadas, foram montadas três placas, em que foram depositados um disco de controle negativo (branco), um disco de controle positivo, contendo $30 \mu \mathrm{g}$ do antibiótico clorafenicol, e três discos com óleo essencial de cravo-da-índia. Na primeira placa, foram colocados discos com $5 \mu \mathrm{L}$ de óleo essencial; na segunda, discos com $10 \mu \mathrm{L}$ e, na terceira, discos com $15 \mu \mathrm{L}$. Após a incubação das placas a $36 \pm 1{ }^{\circ} \mathrm{C}$ durante 48 horas, os resultados foram analisados medindo-se o diâmetro do halo de inibição de crescimento das bactérias, incluindo o diâmetro do disco de papel.

Os resultados foram expressos em milímetros pela média aritmética dos valores dos halos obtidos nas três repetições, sendo as médias comparadas pelo teste de Tukey $(\mathrm{p}<0,05)$, utilizando-se o programa SPSS 10.0.1 Standard Version 1989-1999.

Para determinar a concentração inibitória mínima (CIM), foi utilizado o método indireto de crescimento bacteriano, mediante a densidade ótica em meio de cultura líquido (Maloz, 2005).

Após os resultados obtidos das análises do antibiograma em meio sólido, as 10 bactérias selecionadas foram cultivadas em meio de cultura caldo LB à temperatura de $37^{\circ} \mathrm{C}$ durante 24 horas.

Após o período de crescimento das culturas, foram inoculados, em microtubos $10 \mu \mathrm{L}$ de pré-inóculo $\left(10^{8}\right.$ UFC. $\mathrm{mL}^{-1}$ ), $1 \mathrm{~mL}$ de caldo LB, acrescido de $1 \%$ do emulsificante dimetilsulfóxido (DMSO), contendo diferentes concentrações do óleo essencial. Posteriormente ao processo de inoculação, os microtubos foram incubados em agitador eletromagnético $(60 \mathrm{~Hz})$, por um período de 24 horas, à temperatura de $32^{\circ} \mathrm{C}$.

Antes e após o período de incubação, 0 e 24 horas respectivamente, foram transferidas alíquotas de $100 \mu \mathrm{L}$ da cultura bacteriana para microplacas de fundo chato, realizando-se três leituras para cada concentração do óleo estudado. Para avaliar o crescimento bacteriano (densidade ótica) e para determinar a CIM do óleo essencial sobre determinada bactéria, realizou-se a leitura da microplaca, utilizando-se o leitor automático de microplacas Bio-Tec Instruments Inc., modelo EL800, acoplado em computador com programa Kcjunior, com comprimento de onda pré-selecionado de $490 \mathrm{~nm}$. As concentrações de óleo essencial testadas no experimento e as dez bactérias selecionadas foram de 0,$8 ; 0,75 ; 0,7$; 0,$6 ; 0,5 ; 0,4 ; 0,3 ; 0,2 ; 0,1 \mathrm{mg} \cdot \mathrm{mL}^{-1}$. A inibição do crescimento foi determinada pela diferença entre as leituras realizadas em 24 horas e em 0 hora. Os valores médios de densidade ótica foram analisados estatisticamente por análise de variância, seguida do teste de Tukey $(\mathrm{p}<0,05)$, para determinar a CIM.

\section{Atividade antioxidante pela captura de radicais livres com o teste de DPPH}

A metodologia para avaliação da atividade antioxidante, baseada na medida da extinção da absorção do radical 2,2-difenil-1-picril hidrazil (DPPH) em $515 \mathrm{~nm}$, foi realizada em duplicata, por método espectrofotométrico (Kulisic et al., 2004). A técnica consistiu na incubação, por 10 minutos, de $500 \mu \mathrm{L}$ de uma solução etanólica de DPPH 0,1 mM, com $500 \mu \mathrm{L}$ de soluções, contendo concentrações crescentes de óleo essencial do cravo-da-índia (150; 250; 500; 1000; 2500; 5000; 7500; $10000 \mu \mathrm{g} . \mathrm{mL}^{-1}$ ) em etanol. Procedeu-se da mesma forma para a preparação da solução denominada "controle", substituindo-se, porém, $500 \mu \mathrm{L}$ da amostra por $500 \mu \mathrm{L}$ de solução etanólica do óleo essencial. A solução denominada "branco" foi preparada em concentração 1:1 (v:v), utilizando-se as soluções em diferentes concentrações do óleo essencial e etanol, sem DPPH. O percentual de captação do radical DPPH foi calculado em termos da percentagem de atividade antioxidante (AA\%), conforme a Equação 1.

$$
\mathrm{AA} \%=100-\left\{\left[\left(\text { Abs. }_{\text {amostra }}-\text { Abs. }_{\text {branco }}\right) \times 100\right] \div \text { Abs. }{ }_{\text {controle }}\right\}
$$

A determinação foi feita em espectrofotômetro UVVisível Agilent Technologies, modelo 8453E em comprimento de onda de $515 \mathrm{~nm}$. Após a avaliação da faixa de concentração ideal, calculou-se a concentração de óleo essencial necessária para capturar 50\% do radical livre DPPH $\left(\mathrm{IC}_{50}\right)$ por análise de regressão linear (Carbonari, 2005). 


\section{RESULTADOS E DISCUSSÃO}

\section{Composição química do óleo essencial}

O rendimento de óleo essencial, após extração por hidrodestilação, foi de 1,87\% (m/v). A análise por CG/ EM mostrou o eugenol como composto majoritário (90,3\%), além de $\beta$-cariofileno (4,83\%) e acetato de eugenol (1,87\%).

\section{Atividade antibacteriana}

A partir da Tabela 1, podem-se observar diferenças significativas entre os halos de inibição das bactérias avaliadas; porém, em relação à média destas, os resultados demonstraram que o óleo tem maior atividade inibitória sobre as Gram-positivas.

$\mathrm{O}$ aumento de 5 para $10 \mu \mathrm{L}$ na dose de óleo essencial provocou um aumento significativo de efeito para diversas bactérias, sendo que somente Salmonella choleraesuis mostrou diferenças significativas de sensibilidade nas três concentrações avaliadas, apresentando o menor halo na dose de 5 ì L. Ainda assim, seis bactérias não mostraram acréscimo de sensibilidade com o aumento da dose de óleo essencial. A maior atividade antibacteriana do óleo essencial foi observada sobre Staphylococcus aureus (32,5 mm) em $15 \mu \mathrm{L}$.
De todas as bactérias testadas, apenas Bacillus subtilis e Enterococcus faecalis não apresentaram halo de inibição. Entretanto, Souza et al. (2004) demonstraram a ação inibitória do óleo essencial de Eugenia uniflora sobre Bacillus subtilis.

Estes resultados demonstraram que este método de avaliação pode ser utilizado como método prévio, por ser reconhecido e determinar a sensibilidade de muitos microorganismos a determinadas substâncias, produzindo resultados semiquantitativos. No entanto, ainda é a técnica mais comum para a avaliação de antibacterianos e antifúngicos de óleos essenciais, por ser de fácil execução e requerer pequenas quantidades de amostra (Kalemba \& Kunicka, 2003).

A ação antibacteriana dos componentes dos óleos essenciais pode ocorrer de três formas: pela interferência na dupla camada fosfolipídica da parede celular; pelo aumento da permeabilidade e perda dos constituintes celulares; e por alteração de uma variedade de sistemas enzimáticos, incluindo aqueles envolvidos na produção de energia celular e síntese de componentes estruturais ou por inativação e destruição do material genético (Kalemba \& Kunicka, 2003; Delamare et al., 2007).

Tabela 1. Atividade antibacteriana pelo método de difusão de placas do óleo essencial do cravo-da-índia (Eugenia caryophyllata) sobre bactérias Gram-positivas e Gram-negativas usando-se 5, 10 e $15 \mu \mathrm{L}$ de óleo por disco

\begin{tabular}{|c|c|c|c|}
\hline Bactérias Gram-positivas & $\begin{array}{c}\text { Halo de inibição } \\
5 \mu \mathrm{L}(\mathrm{mm}) *\end{array}$ & $\begin{array}{c}\text { Halo de inibição } \\
10 \mu \mathrm{L}(\mathrm{mm})^{*}\end{array}$ & $\begin{array}{c}\text { Halo de inibição } \\
15 \mu \mathrm{L}(\mathrm{mm}) *\end{array}$ \\
\hline Bacillus subtilis\# & N.S. & N.S. & N.S. \\
\hline Enterococcus faecalis (ATCC 19433) & N.S. & N.S. & N.S. \\
\hline Micrococcus luteus (ATCC10240) & $20,75 \pm 2,21^{\mathrm{a}}$ & $23,75 \pm 0,95^{\mathrm{a}}$ & $24,00 \pm 1,82^{\mathrm{a}}$ \\
\hline Sarcina sp.\# & $16,50 \pm 2,38^{a}$ & $26,50 \pm 1,29^{b}$ & $27,50 \pm 1,29^{b}$ \\
\hline Staphylococcus aureus (ATCC 6538) & $31,50 \pm 2,38^{a}$ & $32,00 \pm 0,81^{\mathrm{a}}$ & $32,50 \pm 1,29^{a}$ \\
\hline S. epidermidis (ATCC 12228) & $21,50 \pm 1,29^{a}$ & $23,75 \pm 0,95^{\mathrm{b}}$ & $24,25 \pm 0,95^{\mathrm{b}}$ \\
\hline Streptococcus mutans (ATCC 5175) & $18,50 \pm 0,57^{\mathrm{a}}$ & $22,50 \pm 1,73^{\mathrm{b}}$ & $22,50 \pm 2,08^{\mathrm{b}}$ \\
\hline Média & 21,75 & 25,70 & 26,15 \\
\hline Bactérias Gram-negativas & $\begin{array}{c}\text { Halo de inibição } \\
5 \mu \mathrm{L}(\mathrm{mm})^{*}\end{array}$ & $\begin{array}{c}\text { Halo de inibição } \\
10 \mu \mathrm{L}(\mathrm{mm})^{*}\end{array}$ & $\begin{array}{c}\text { Halo de inibição } \\
15 \mu \mathrm{L}(\mathrm{mm})^{*}\end{array}$ \\
\hline Acinetobacter sp.\# & $13,50 \pm 1,0^{\mathrm{a}}$ & $17,50 \pm 1,29^{\mathrm{b}}$ & $19,00 \pm 2,94^{b}$ \\
\hline Aeromonas sp.\# & $15,00 \pm 0,81^{\mathrm{a}}$ & $15,75 \pm 0,50^{\mathrm{a}}$ & $16,50 \pm 1,73^{a}$ \\
\hline Citrobacter freundii (ATCC 8090) & $17,00 \pm 3,91^{\mathrm{a}}$ & $23,50 \pm 1,29^{\mathrm{ab}}$ & $26,25 \pm 5,96^{b}$ \\
\hline Enterobacter cloacae\# & $17,00 \pm 1,82^{\mathrm{a}}$ & $19,00 \pm 1,82^{\mathrm{a}}$ & $23,75 \pm 2,21^{b}$ \\
\hline Escherichia coli (ATCC 25922) & $19,00 \pm 1,63^{\mathrm{a}}$ & $19,75 \pm 0,95^{\mathrm{a}}$ & $20,50 \pm 1,29^{\mathrm{a}}$ \\
\hline Proteus vulgaris (ATCC 13315) & $13,50 \pm 1,26^{\mathrm{a}}$ & $15,75 \pm 0,95^{\mathrm{a}}$ & $21,25 \pm 1,90^{\mathrm{b}}$ \\
\hline Pseudomonas aeruginosa (ATCC 27853) & $13,00 \pm 1,63^{\mathrm{a}}$ & $14,00 \pm 0,81^{\mathrm{a}}$ & $15,50 \pm 1,29^{a}$ \\
\hline Salmonella choleraesuis (ATCC 10708) & $11,25 \pm 0,95^{\mathrm{a}}$ & $15,75 \pm 0,95^{b}$ & $18,25 \pm 0,95^{c}$ \\
\hline Serratia sp. (ATCC 13880) & $16,50 \pm 2,38^{a}$ & $26,50 \pm 1,29^{\mathrm{b}}$ & $27,50 \pm 1,29^{\mathrm{b}}$ \\
\hline Shigella flexneri (ATCC 12022) & $13,25 \pm 1,25^{a}$ & $15,75 \pm 0,95^{\mathrm{a}}$ & $19,75 \pm 1,70^{\mathrm{b}}$ \\
\hline Xanthomonas campestris\# & $15,75 \pm 0,50^{\mathrm{a}}$ & $18,25 \pm 1,50^{\mathrm{a}}$ & $18,75 \pm 2,21^{\mathrm{a}}$ \\
\hline Média & 14,98 & 18,32 & 20,64 \\
\hline
\end{tabular}

N. S. = Não sensível; \# Micro-organismos obtidos a partir do Instituto Biológico, Campinas, São Paulo; ATCC: American Type Culture Collection (USA); * Médias seguidas da mesma letra na mesma linha, não diferem entre si pelo teste de Tukey, a 5\% de probabilidade. 
Tabela 2. Concentrações inibitórias mínimas (CIM) do óleo essencial de cravo-da-índia (Eugenia caryophyllata)

\begin{tabular}{lc}
\hline Bactérias Gram-positivas & CIM (mg.mL-1 \\
\hline Staphylococcus epidermidis & 0,2 \\
Staphylococcus aureus & 0,3 \\
Sarcina sp. & 0,4 \\
Micrococcus luteus & 0,6 \\
Streptococcus mutans & 0,6 \\
Média & 0,5 \\
\hline Bactérias Gram-negativas & CIM (mg. $\left.\mathbf{m L}^{-1}\right)$ \\
\hline Enterobacter cloacae & 0,5 \\
Salmonella choleraesuis & 0,5 \\
Serratia sp. & 0,5 \\
Shigella flexneri & 0,6 \\
Pseudomonas aeruginosa & 0,8 \\
Média & 0,58 \\
\hline
\end{tabular}

Na avaliação dos resultados das CIM, apresentados na Tabela 2, observou-se que todos as bactérias apresentaram-se susceptíveis ao óleo essencial de Eugenia caryophyllata.

As CIM das bactérias Gram-positivas apresentaram variação de $0,2 \mathrm{mg} \cdot \mathrm{mL}^{-1}$ (Staphylococcus aureus) a 0,6 mg. $\mathrm{mL}^{-1}$ (Streptococcus mutans). Já a variação das CIM das bactérias Gram-negativas foi de 0,5 mg.mL $L^{-1}$ (Salmonella choleraesuis, Enterobacter cloacae e Serratia sp.) a $0,8 \mathrm{mg} \cdot \mathrm{mL}^{-1}$ (Pseudomonas aeruginosa).

Os resultados de CIM são da mesma ordem de magnitude dos resultados encontrados com óleo essencial de Origanum majorana (Busatta et al., 2008), Thymus vulgaris L. (Kalpoutzakis et al., 2001), Salvia sp. (Tepe et al., 2004), Melissa officinalis (Mimica-Dukic et al., 2004) e Origanum vulgare L. (Busatta et al., 2007).

Para Nascimento et al. (2007), os métodos de atividade antimicrobiana (diluição e difusão) não são necessariamente comparáveis. Isto por que o método de diluição mostra ser o que melhor disponibiliza dados quantitativos, enquanto a difusão em placa constitui-se um método qualitativo.

Existem fatores que podem interferir nos valores da CIM, obtidos por meio de métodos de difusão e diluição: condições de cultivo (tempo de incubação, temperatura, taxa de oxigênio), meio de cultura, concentração das substâncias testadas, dispersão e emulsificação dos agentes utilizados na emulsão óleo-água (Rios \& Recio, 2005).

\section{Atividade antioxidante pela captura de radicais livres com o teste de DPPH}

O DPPH é um radical livre, estável em temperatura ambiente, que produz uma solução violeta em etanol. Na presença de componentes antioxidantes, o DPPH é reduzido, produzindo uma solução etanólica transparente.
Tabela 3. Percentagem da neutralização do DPPH do óleo essencial do cravo-da-índia (Eugenia caryophyllata)

\begin{tabular}{lc}
\hline Concentrações $\left(\boldsymbol{\mu g} \cdot \mathbf{m L}^{-1}\right)$ & Atividade Antioxidante (\%) \\
\hline 150 & 44,1 \\
250 & 43,22 \\
500 & 45,27 \\
1000 & 40,88 \\
2500 & 70,33 \\
5000 & 73,7 \\
7500 & 90,1 \\
10000 & 95,6 \\
\hline
\end{tabular}

Os resultados, obtidos após a determinação da atividade antioxidante do óleo essencial em diferentes concentrações, estão representados na Tabela 3 e demonstraram que o percentual antioxidante aumentou proporcionalmente com a concentração de óleo adicionado, atingindo o valor máximo de 95,6\% de atividade antioxidante para a concentração de $10000 \mu \mathrm{g} \cdot \mathrm{mL}^{-1}$.

A correlação entre a atividade antioxidante (\%) e a concentração de óleo utilizado ( $\mathrm{Y}=0,0057 \mathrm{x}+43,623)$, com $\mathrm{R}^{2}=0,9225$, forneceu um $\mathrm{IC}_{50}$ de $1.118,77 \mu \mathrm{g} \cdot \mathrm{mL}^{-1}$, que é a concentração de óleo essencial necessária para causar $50 \%$ de atividade antioxidante (Figura 1). Essa concentração é alta, se comparada com antioxidantes comerciais como o ácido ascórbico $\left(\mathrm{IC}_{50}=2,15 \mu \mathrm{g} \cdot \mathrm{mL}^{-1}\right)$ e o BHT $\left(\mathrm{IC}_{50}=5,37 \mu \mathrm{g} \cdot \mathrm{mL}^{-1}\right)$.

Segundo Mensor et al. (2001), o IC I0 $_{\text {de } 38,91 ~ \mu g . \mathrm{mL}^{-1}}$ do extrato vegetal de Ginkgo biloba L. qualifica-o como um extrato de alta atividade antioxidante, pois apresenta a mesma magnitude que o $\mathrm{IC}_{50}$ de diversas plantas reconhecidamente antioxidantes como a erva-mate (Ilex paraguariensis A. St.-Hil.). Isso demonstra que o óleo essencial de Eugenia caryophyllata apresenta uma baixa atividade antioxidante quando comparado com o extrato de G. biloba, mas que pode ser considerada superior ao encontrada em outros óleos essenciais, indicando um potencial uso em produtos alimentícios. Neste sentido, diversos autores avaliaram a atividade antioxidante de óleos essenciais de algumas plantas e encontraram os seguintes resultados: Hippomarathrum microcarpum Petrov com $\mathrm{IC}_{50}$ de $10.690 \mu \mathrm{g} . \mathrm{mL}^{-1}$, Chaerophyllum libanoticum Boiss. \& Kotschy com IC $_{50}$ superior a $30.000 \mu \mathrm{g} . \mathrm{L}^{-1}$, Rosmarinus officinalis com $\mathrm{IC}_{50}$ de $20.000 \mu \mathrm{g} . \mathrm{mL}^{-1}$, Artemisia fragrans Willd. com $\mathrm{IC}_{50}$ de $7.860 \mu \mathrm{g} . \mathrm{mL}^{-1}$, Cinnamomum camphora (L.) J. Presl com $\mathrm{IC}_{50}$ de $12.942 \mu \mathrm{g} \cdot \mathrm{mL}^{-1}$, Artemisia austriaca Jacq. com IC IC $_{50}$ de $8.060 \mu \mathrm{g} . \mathrm{mL}^{-1}$ e Petroselinum crispum (Mill.) Fuss com $\mathrm{IC}_{50}$ de $80.210 \mu \mathrm{g} \cdot \mathrm{mL}^{-1}$ (Özer et al., 2007). $\mathrm{O}$ efeito antioxidante observado se deve à sua composição química, composta majoritariamente por eugenol, o qual possui reconhecida atividade antioxidante (Lee et al., 2005). 


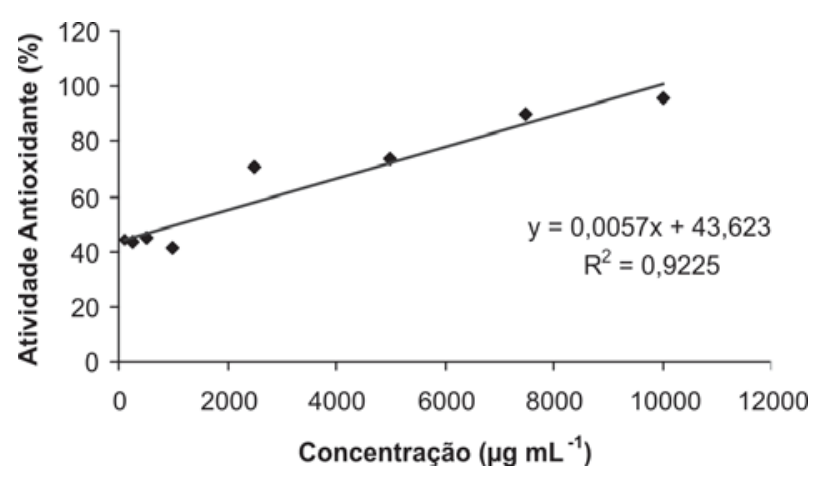

Figura 1. Curva de calibração da atividade antioxidante do óleo essencial do cravo-da-índia (Eugenia caryophyllata).

\section{CONCLUSÕES}

O óleo essencial do cravo-da-índia tem como composto majoritário o eugenol.

As concentrações inibitórias mínimas (CIM) do óleo essencial de cravo-da-índia variaram de $0,2 \mathrm{mg} \cdot \mathrm{mL}^{-1}$ a 0,6 mg.mL ${ }^{-1}$ para as bactérias Gram-positivas e de $0,5 \mathrm{mg} \cdot \mathrm{mL}^{-1} \mathrm{a}$ 0,8 $\mathrm{mg}^{\mathrm{ml}} \mathrm{m}^{-1}$ para as bactérias Gram-negativas.

A atividade antioxidante apresenta uma correlação linear com a concentração de óleo essencial (95,6\% para concentração de $10.000 \mu \mathrm{g} \cdot \mathrm{mL}^{-1}$ ).

Os resultados mostram que o óleo essencial do cravoda-índia tem potencial como antioxidante, constituindose uma boa opção para a formulação de novos produtos alimentícios.

\section{REFERENCIAS}

Busatta C, Mossi AJ, Rodrigues MRA, Cansian RL \& Oliveira JV (2007) Evaluation of Origanum vulgare essential oil as antimicrobial agent in sausage. Brazilian Journal of Microbiology, 38:610-616.

Busatta C, Vidal RS, Popiolski AS, Mossi AJ, Dariva C, Rodrigues MRA, Corazza FC, Corazza ML, Oliveira JV \& Cansian RL (2008) Application of Origanum majorana L. essential oil as antimicrobial agent in sausage. Food Microbiology, 25:207-211.

Carbonari KA (2005) Avaliação do potencial antioxidante (in vitro e in vivo) e antiinflamatório de Ouratea parviflora, Polymnia sonchifolia e Marlierea obscura. Dissertação de Mestrado. Universidade Federal de Santa Catarina, Florianópolis. 132p.

Celiktas OY, Kocabas EEH, Bedir E, Sukan FV, Ozek T \& Baser KHC (2007) Antimicrobial activity of methanol extracts and essential oils of Rosmarinus officinalis, depending on location and seasonal variations. Food Chemistry, 100:553-559.

Delamare AP, Pistorello ITM, Artico L, Serafini LA \& Echeverrigaray S (2007) Antibacterial activity of essential oils of Salvia officinalis L. and Salvia triloba L. cultivated in South Brazil. Food Chemistry, 100:603-608.

Kalemba D \& Kunicka A (2003) Antibacterial and antifungal properties of essential oils. Current Medicinal Chemistry, 10:813-829.

Kalpoutzakis E, Aligiannis N, Mitaku S \& Charvala C (2001) Composition of the essential oil of two nepeta especies and in vitro evaluation of their activity against Helicobacter pylori. Plants Medicinals, 67:880-883.
Kulisic T, Radonic A, Katalinic V \& Milos M (2004) Use of different methods for testing antioxidative activity of oregano essential oil. Food Chemistry, 85:633-640.

Lee SJ, Umano K, Shibamoto T \& Lee KG (2005) Identification of volatile components in basil (Ocimum basilicum L.) and thyme leaves (Thymus vulgaris L.) and their antioxidant properties. Food Chemistry, 91:131-137.

Maloz MKP (2005) Caracterização morfológica, avaliação agronômica, química e da atividade antimicrobiana do óleo essencial de diferentes espécies exóticas de gênero Salvia. Dissertação de Mestrado. Universidade de Caxias do Sul, Caxias do Sul. 87p.

Mau JL, Chen C-P \& Hsieh PC (2001) Antimicrobial effects of extracts from Chinese chive, cinnamon and corn fructus. Journal of Agricultural and Food Chemistry, 49:183-188.

Mensor LL, Menezes FS, Leitão GG, Reis AS, Santos TC, Coube CS \& Leitão SG (2001) Screnning of Brazilian plant extracts for antioxidant activiy by the use of DPPH free radical method. Phytotherapy Research, 53:127-130.

Mimica-Dukic N, Bozin B, Socovic M \& Simin N (2004) Antimicrobial and antioxidant of Melissa officinalis L. (Lamiaceae) essential oil. Journal of Agricultural and Food Chemistry, 52:2485-2489.

Moreira MR, Ponce AG, Del Valle CE \& Roura SI (2005) Inhibitory parameters of essential oils to reduce a foodborne pathogen. LWT-Food Science and Technology, 38:565-570.

Nascimento GGF, Locatelli J \& Freitas PC (2000) Antibacterial activity of plant extracts and phytochemicals on antibiotic resistant bacteria. Brazilian Journal of Microbiology, 31:247256.

Nascimento PFC, Nascimento AC, Rodrigues CS, Antoniolli AR, Santos PO, Júnior BMA \& Trindade RC (2007) Atividade antimicrobiana dos óleos essenciais: uma abordagem multifatorial dos métodos. Revista Brasileira de Farmacognosia, 17:108-113.

Özer H, Sökmen M, Güllüce M \& Adigüzel A (2007) Chemical composition and antimicrobial and antioxidant activities of the essential oil and methanol extract of Hippomarathrum microcarpum (Bieb.) from Turkey. Journal of Agricultural and Food Chemistry, 55:937-942.

Rios JL \& Recio MC (2005) Medicinal plants and antimicrobial activity. Journal of Ethnopharmacology, 100:80-84.

Semwal AD, Sharma GK \& Arya SS (1997) Antoxygenic activity of turmeric (Curcuna longa) in sunflower oil and ghee. Journal of Food Science and Technology, 34:67-69.

Souza EL, Lima EO \& Narain N (2003) Especiarias: uma alternativa para o controle de qualidade sanitária e de vida útil de alimentos, frente às novas perspectivas da indústria alimentícia. Higiene Alimentar, 17:38-42.

Souza GC, Haas APS, Poser GL, Schapoval EES \& Elisabetsky E (2004) Ethnopharmacological studies of antimicrobial remedies in the south of Brazil. Journal of Ethnopharmacology, 90:135143.

Tepe B, Donney E, Unlu M, Candan F, Daferera D, Unlu GV, Polissiou M \& Sokmen AA (2004) Antimicrobial and antioxidative activities of the essential oils and methanol extracts of S. cryptanha (Montbret et Aucher ex Benth) S. multicaulis (Vahl). Food Chemistry, 84:519-525. 